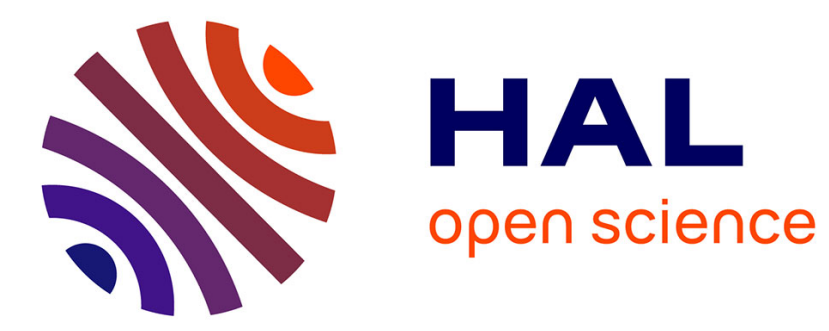

\title{
Stabilization of MISO fractional systems with delays
}

Le Ha Vy Nguyen, Catherine Bonnet

\section{To cite this version:}

Le Ha Vy Nguyen, Catherine Bonnet. Stabilization of MISO fractional systems with delays. Automatica, 2017, 83, pp.337 - 344. 10.1016/j.automatica.2017.06.032 . hal-01627128

\section{HAL Id: hal-01627128 \\ https://inria.hal.science/hal-01627128}

Submitted on 21 Jan 2018

HAL is a multi-disciplinary open access archive for the deposit and dissemination of scientific research documents, whether they are published or not. The documents may come from teaching and research institutions in France or abroad, or from public or private research centers.
L'archive ouverte pluridisciplinaire HAL, est destinée au dépôt et à la diffusion de documents scientifiques de niveau recherche, publiés ou non, émanant des établissements d'enseignement et de recherche français ou étrangers, des laboratoires publics ou privés. 


\title{
Stabilization of MISO fractional systems with delays *
}

\author{
Le Ha Vy Nguyen ${ }^{a}$, Catherine Bonnet ${ }^{\mathrm{a}}$, \\ anria, Université Paris-Saclay, L2S-CentraleSupélec, 3 Rue Joliot-Curie, 91192 Gif-sur-Yvette, France
}

\begin{abstract}
We consider multi-input single-output (MISO) fractional systems of commensurate fractional orders with different input or output delays. We derive explicit expressions of left and right coprime factorizations over $H_{\infty}$ and of the associated Bézout factors of the transfer matrix of the systems. These factors allow the construction of the Youla-Kučera parametrization of the set of stabilizing controllers which guarantee the internal stability of the closed-loop systems.
\end{abstract}

Key words: Coprime factorization; H-infinity; stabilizing controllers; time delay; MIMO.

\section{Introduction}

Fractional systems are systems described by differential equations involving non-integer order derivatives and/or integrals. Consequently, in the frequency domain, their transfer functions contain non-integer powers of the Laplace variable $s$. This kind of models has become more popular in many fields in the past two decades since it provides a better fit to data being then more succinct than a standard model. Refer, for example, to [7] for basic backgrounds on fractional calculus and to $[17,5]$ for its recent applications on modeling.

Delays are encountered almost everywhere due, for example, to distance of transmission and it is well-known that they have important effects on the stability of the systems [16].

While integer-order systems with delays have been intensively studied [16], the literature on fractional systems with delays is still quite small. Particularly, the stabilization problem of fractional systems with delays has rarely been addressed. Some available studies are classical [13] and fractional PID controller design [6], fractional sliding mode control [18], factorization approach to control synthesis $[1,3]$.

In the framework of fractional representation approach to synthesis problems [20], SISO fractional delay systems was considered in $[1,3]$ and coprime factorizations

\footnotetext{
^ Corresponding author L. H. V. Nguyen.

Email addresses: nguyenlehavy@gmail.com (Le Ha Vy Nguyen), catherine.bonnet@inria.fr (Catherine Bonnet).
}

together with the corresponding Bézout factors of the transfer function of these systems have been derived. In [4], coprime factors were presented for a large class of MIMO infinite-dimensional systems which include delay systems. The factors were determined from a state-space realization of the (regular) system which was given in terms of the semigroup of the system. Such realizations are not much considered for fractional systems.

For the particular class of MIMO (integer-order) systems with I/O delays, the problem of parametrization of stabilizing controllers was solved in $[8,9]$. The idea was to reduce the problem to an equivalent finite-dimensional stabilization problem by involving an unstable finitedimensional system and a stable infinite-dimensional system (FIR filter). In [10], a procedure to compute right coprime factorizations over a Bézout domain was proposed for spectrally controllable MIMO (integer-order) systems with input delays. For MISO structure, a class of (integer-order) systems with multiple transmission delays was studied in [2] and coprime factorizations and associated Bézout factors over $H_{\infty}$ were derived.

In this paper, we are interested in the stabilization problem of MISO fractional systems with different I/O delays which are not necessarily commensurate. This MISO structure appeared, for example, in communication systems [15]. We would like to obtain the set of all stabilizing controllers by determining a doubly coprime factorization over $H_{\infty}$ of the transfer matrix and the associated Bézout factors, which allow the construction of the Youla-Kučera parametrization [20]. As in the finitedimensional case, the Youla-Kučera parametrization gives the set of all $H_{\infty}$-stabilizing controllers in terms of one free parameter. Note that in [14], a parametriza- 
tion of the set of all stabilizing controllers is given in terms of two free parameters for MIMO systems once we already know a particular stabilizing controller. Our strategy here is to work directly on the Bézout identity in order to get explicit expressions of Bézout factors in terms of the matrix transfer function. Such explicit expressions could not be easily derived in $[8,9,10]$ even in the case of standard delay systems. We hope that the explicit form will facilitate the use of these factors in controllers design while the use of the frequency domain representation of the systems agrees well with the modeling practice of fractional systems [17].

The paper is organized as follows. In Section 2, the class of systems of interest and some background are presented. The results are stated in Sections 3 and 4. We give in Section 3 explicit expressions of left coprime factorizations and associated Bézout factors over $H_{\infty}$ of the transfer function of the systems under study. Right coprime factorizations and right Bézout factors are given in Section 4 for a large subclass of the class of systems considered. Examples are provided to illustrate the results. Finally, Section 5 gives conclusions and perspectives.

\section{A class of MISO fractional time-delay systems}

We consider systems described by transfer matrices of the form

$$
G(s)=\left[e^{-s h_{1}} R_{1}\left(s^{\alpha}\right), \ldots, e^{-s h_{n}} R_{n}\left(s^{\alpha}\right)\right],
$$

where $0 \leq h_{k} \in \mathbb{R}$ for $k=1, \ldots, n$ are the delays; $\alpha \in \mathbb{R}, 0<\alpha<1 ; R_{k}\left(s^{\alpha}\right)=\widetilde{q}_{k}\left(s^{\alpha}\right) / \widetilde{p}_{k}\left(s^{\alpha}\right)$, where $\widetilde{p}_{k}$ and $\widetilde{q}_{k}$ are polynomials of integer degree in $s^{\alpha}, \widetilde{p}_{k}\left(s^{\alpha}\right)$ and $\widetilde{q}_{k}\left(s^{\alpha}\right)$ have no common roots, and $\operatorname{deg} \widetilde{p}_{k} \geq \operatorname{deg} \widetilde{q}_{k}$ for $k=1, \ldots, n ; d_{k}$ is the degree in $s^{\alpha}$ of $\widetilde{p}_{k} ; s$ is in the principle branch $\mathbb{C} \backslash \mathbb{R}_{-}$, that is $\arg (s) \in(-\pi, \pi)$, in order to guarantee a unique value of the transfer function involving $s^{\alpha}$ with $\alpha \in(0,1)$.

Some notations used are $\mathbb{C}_{+}=\{s \in \mathbb{C} \mid \operatorname{Re}(s)>0\}$, $\mathbb{Z}_{+}=\{p \in \mathbb{Z} \mid p>0\}, \overline{\mathbb{Z}_{+}}=\{p \in \mathbb{Z} \mid p \geq 0\}$.

We are interested in $H_{\infty}$-stability, i.e. a SISO system is stable if its transfer function $K(s)$ belongs to the $H_{\infty}$ space of analytic and bounded functions in $\mathbb{C}_{+}$with $\|K\|_{H_{\infty}}=\sup _{s \in \mathbb{C}_{+}}|K(s)|$. Let us denote $\mathbf{M}\left(H_{\infty}\right)$ the set of matrices whose components are in $H_{\infty}$.

The following notion of coprimeness is considered.

A system $G$ is said to have a right coprime factorization (r.c.f.) $(N, M)$ over $H_{\infty}$ if $G=N M^{-1}$, $\operatorname{det} M \neq 0, N$, $M \in \mathbf{M}\left(H_{\infty}\right)$ and there exist $X, Y \in \mathbf{M}\left(H_{\infty}\right)$ such that $X M+Y N=I$. Then $X, Y$ are called right Bézout factors.
A system $G$ is said to have a left coprime factorization (l.c.f.) $(\widetilde{M}, \widetilde{N})$ over $H_{\infty}$ if $G=\widetilde{M}^{-1} \widetilde{N}$, $\operatorname{det} \widetilde{M} \neq 0, \widetilde{M}$, $\widetilde{N} \in \mathbf{M}\left(H_{\infty}\right)$ and there exist $\widetilde{X}, \widetilde{Y} \in \mathbf{M}\left(H_{\infty}\right)$ such that $\widetilde{M} \widetilde{X}+\widetilde{N} \widetilde{Y}=I$. Then $\widetilde{X}, \widetilde{Y}$ are called left Bézout factors.

For $\widetilde{M}, \widetilde{N} \in \mathbf{M}\left(H_{\infty}\right)$, there exist $\widetilde{X}, \widetilde{Y} \in \mathbf{M}\left(H_{\infty}\right)$ such that $\widetilde{M} \widetilde{X}+\widetilde{N} \widetilde{Y}=I$ if and only if $\inf _{s \in \mathbb{C}_{+}} \sigma_{m}\left([\widetilde{M}, \widetilde{N}]^{T}\right)>$ 0 , where $\sigma_{m}(\cdot)$ is the smallest singular value of a matrix [20, Lemma 8.1.13 and Example 8.1.15].

If $G$ has an r.c.f. $(N, M)$ and an l.c.f. $(\widetilde{M}, \widetilde{N})$, then the set of all controllers guaranteeing the internal stability of the closed-loop system is given by the Youla-Kučera parametrization

$$
\begin{aligned}
C(G)= & \left\{(X-R \widetilde{N})^{-1}(Y+R \widetilde{M}) \mid R \in \mathbf{M}\left(H_{\infty}\right),\right. \\
& \operatorname{det}(X-R \widetilde{N}) \neq 0\} \\
= & \left\{(\widetilde{Y}+M R)(\widetilde{X}-N R)^{-1} \mid R \in \mathbf{M}\left(H_{\infty}\right),\right. \\
& \operatorname{det}(\widetilde{X}-N R) \neq 0\},
\end{aligned}
$$

where $X, Y$ and $\tilde{X}, \tilde{Y}$ are respectively the corresponding right and left Bézout factors [20]. For $R=0$, we obtain two particular stabilizing controllers $C=X^{-1} Y$ and $C=\widetilde{Y} \widetilde{X}^{-1}$.

Poles (resp. roots) in the closed right half-plane $\overline{\mathbb{C}_{+}}$are referred to as unstable poles (resp. roots).

The following notations will be of intense use later.

Denote $p\left(s^{\alpha}\right)$ the lowest common denominator of $R_{k}\left(s^{\alpha}\right)$ for $k=1, \ldots, n ; d$ the degree in $s^{\alpha}$ of $p\left(s^{\alpha}\right)$. Then rational transfer functions $R_{k}\left(s^{\alpha}\right)$ can be rewritten as

$$
R_{k}\left(s^{\alpha}\right)=\frac{q_{k}\left(s^{\alpha}\right)}{p\left(s^{\alpha}\right)}
$$

where $q_{k}$ are polynomials in $s^{\alpha}$.

We can decompose

$$
p\left(s^{\alpha}\right)=\left(s^{\alpha}\right)^{m_{0}}\left(\prod_{i=1}^{N}\left(s^{\alpha}-b_{i}\right)^{m_{i}}\right)\left(\prod_{j=1}^{N^{\prime}}\left(s^{\alpha}-c_{j}\right)^{m_{j}^{\prime}}\right),
$$

where $b_{i} \in \mathcal{D}:=\{\sigma \in \mathbb{C} \backslash\{0\} \mid-\pi \alpha / 2 \leq \operatorname{Arg}(\sigma) \leq$ $\pi \alpha / 2\} ; c_{j} \in \mathbb{C} \backslash\{\mathcal{D} \cup\{0\}\} ; m_{0} \in \overline{\mathbb{Z}_{+}}, m_{i}, m_{j}^{\prime} \in \overline{\mathbb{Z}_{+}}$for $i=1, \ldots, N$ and $j=1, \ldots, N^{\prime}$. Hence $s_{i}=b_{i}^{1 / \alpha}$ are the non-zero unstable roots in $s$ of $p\left(s^{\alpha}\right)$.

Similarly, we write

$\widetilde{p}_{k}\left(s^{\alpha}\right)=\left(s^{\alpha}\right)^{m_{0 k}}\left(\prod_{i=1}^{N}\left(s^{\alpha}-b_{i}\right)^{m_{i k}}\right)\left(\prod_{j=1}^{N^{\prime}}\left(s^{\alpha}-c_{j}\right)^{m_{j k}^{\prime}}\right)$, 
where $m_{0 k}, m_{i k}, m_{j k}^{\prime} \in \overline{\mathbb{Z}_{+}}$for $i=1, \ldots, N$, $j=1, \ldots, N^{\prime}$ and $k=1, \ldots, n$. It is obvious that $m_{0 k} \leq m_{0}, m_{i k} \leq m_{i}$, and $m_{j k}^{\prime} \leq m_{j}^{\prime}$.

\section{Left coprime factorizations and Bézout fac- tors}

In this section, we present l.c.f.'s and Bézout factors for the transfer matrix (1).

\subsection{Left coprime factorizations}

Due to the dimension of the transfer matrix, finding an l.c.f. is straightforward. The next proposition was presented in [11] and is recalled here together with its proof for the paper to be self-contained.

Proposition 1 ([11]) Let $G(s)$ be given by (1). Then $(\widetilde{M}(s), \widetilde{N}(s))$, where

$$
\begin{aligned}
\widetilde{M}(s) & =\frac{p\left(s^{\alpha}\right)}{\left(s^{\alpha}+1\right)^{d}}, \\
\widetilde{N}(s) & =\frac{1}{\left(s^{\alpha}+1\right)^{d}}\left[e^{-s h_{1}} q_{1}\left(s^{\alpha}\right), \ldots, e^{-s h_{n}} q_{n}\left(s^{\alpha}\right)\right],
\end{aligned}
$$

is an l.c.f. over $H_{\infty}$ of $G(s)$.

PROOF. It is obvious that $\widetilde{M}(s)^{-1} \widetilde{N}(s)=G(s)$.

We see that $\widetilde{M}(s) \in H_{\infty}$. Also, each component of $\widetilde{N}(s)$ is in $H_{\infty}$, and then $\tilde{N}(s) \in \mathbf{M}\left(H_{\infty}\right)$.

For all roots $\sigma$ of $p\left(s^{\alpha}\right)$, there exists at least one $1 \leq k \leq$ $n$ such that $q_{k}(\sigma) \neq 0$. Thusinf $\operatorname{siC}_{+}\left(\sum_{k=1}^{n}\left|\widetilde{N}_{k}\right|+|\widetilde{\widetilde{M}}|\right)>$ 0 which ensures that $(\widetilde{M}, \widetilde{N})$ is an l.c.f. over $H_{\infty}$ of $G$.

\subsection{Bézout factors}

Now we propose left Bézout factors corresponding to the l.c.f. obtained above. From the left Bézout identity, we derive that $\widetilde{X}=\widetilde{M}^{-1}(1-\tilde{N} \widetilde{Y})$. The idea is to choose $\tilde{Y} \in \mathbf{M}\left(H_{\infty}\right)$ such that $\tilde{X} \in H_{\infty}$. To achieve that, we interpolate $(1-\tilde{N} \widetilde{Y})$ at unstable zeros of $\widetilde{M}$.

For some simple classes of systems (1), Bézout factors were derived in [11]. In this paper, we consider the most general case of systems (1) where techniques developed in $[2,11]$ are no longer applicable and we need here to include non-commensurate powers of $s$ in $\widetilde{X}$ and $\widetilde{Y}$.
First, let us denote

$k_{0}:=\min \left\{k \mid k \in\{1, \ldots, n\}, m_{0 k}=m_{0}\right\}$,

$k_{i}:=\min \left\{k \mid k \in\{1, \ldots, n\}, m_{i k}=m_{i}\right\}, \quad i=1, \ldots, N$,

$f_{k}:=\sum_{i \in\{1, \ldots, N\}: k_{i}=k} m_{i}, \quad k=1, \ldots, n$,

$\mathcal{L}\left(m_{0}, \alpha\right):=\left\{x \in \mathbb{R} \mid x=a+b \alpha<m_{0} \alpha, a, b \in \overline{\mathbb{Z}_{+}}\right\}$.

The latter set will be of use to describe the power in $s$ of the product $s^{a}\left(s^{\alpha}\right)^{b}$ with $a, b \in \overline{\mathbb{Z}_{+}}$.

Proposition 2 Let $G(s)$ be given by (1). Then Bézout factors corresponding to the l.c.f. (2) are given by

$$
\begin{aligned}
& \tilde{X}(s)=\frac{\left(s^{\alpha}+1\right)^{d} u\left(s^{\alpha}\right)-\sum_{k=1}^{n} e^{-s h_{k}} q_{k}\left(s^{\alpha}\right) \mu_{k}(s)}{p\left(s^{\alpha}\right) u\left(s^{\alpha}\right)}, \\
& \widetilde{Y}(s)=\left[\frac{\mu_{1}(s)}{u\left(s^{\alpha}\right)}, \ldots, \frac{\mu_{n}(s)}{u\left(s^{\alpha}\right)}\right]^{T},
\end{aligned}
$$

where $u\left(s^{\alpha}\right)$ is a polynomial in $s^{\alpha}$ of degree greater or equal to $d$ whose zeros are stable, and for $k=1, \ldots, n$ the polynomials $\mu_{k}(s)$ have the following form

$\mu_{k}(s)= \begin{cases}\sum_{\lambda \in \mathcal{L}\left(m_{0}, \alpha\right)} \beta_{\lambda k} s^{\lambda}+\sum_{j=m_{0}}^{m_{0}+f_{k}-1} \beta_{(j \alpha) k}\left(s^{\alpha}\right)^{j} & \text { if } k=k_{0}, \\ \sum_{j=0}^{f_{k}-1} \beta_{(j \alpha) k}\left(s^{\alpha}\right)^{j} & \text { if } k \neq k_{0},\end{cases}$

and satisfy

$$
\left[\left(s^{\alpha}+1\right)^{d} u\left(s^{\alpha}\right)-\sum_{k=1}^{n} e^{-s h_{k}} q_{k}\left(s^{\alpha}\right) \mu_{k}(s)\right]=O\left(s^{m_{0} \alpha}\right)
$$

as $s \rightarrow 0$ and

$$
\left[\left(s^{\alpha}+1\right)^{d} u\left(s^{\alpha}\right)-\sum_{k=1}^{n} e^{-s h_{k}} q_{k}\left(s^{\alpha}\right) \mu_{k}(s)\right]^{(l)}=0
$$

for each non-zero unstable root $s=b_{i}^{1 / \alpha}, i=1, \ldots, N$, of $p\left(s^{\alpha}\right)$ and for $0 \leq l \leq m_{i}-1$.

Remark 1 If $f_{k}=0$, then $\mu_{k}(s)=\sum_{\lambda \in \mathcal{L}\left(m_{0}, \alpha\right)} \beta_{\lambda k} s^{\lambda}$ when $k=k_{0}$ and $\mu_{k}(s)=0$ otherwise.

PROOF. It is easy to verify that $\widetilde{X}(s)$ and $\widetilde{Y}(s)$ satisfy the left Bézout identity.

The degree of $\mu_{k}(s)$ is smaller than or equal to the degree of $u\left(s^{\alpha}\right)$, and so $\widetilde{Y}(s) \in \mathbf{M}\left(H_{\infty}\right)$. 
We see that $\tilde{X}(s)$ is bounded at $\infty$ in $\mathbb{C}_{+}$. Moreover, due to $(6)$, the numerator of $\widetilde{X}(s)$ has the same non-zero unstable roots as the denominator, which assures that $\widetilde{X}(s)$ is analytic in $\mathbb{C}_{+}$. The boundedness of the function at $s=0$ is satisfied by $(5)$. Then $\widetilde{X}(s) \in H_{\infty}$.

Now it remains to prove the existence of $\mu_{k}(s)$ satisfying the two conditions (5) and (6). The details are reported in Appendix A.

Remark 2 If $m_{0} \alpha \leq 1$ (this case has been considered in [11]) or $\alpha=1 / m$ with $m \in \overline{\mathbb{Z}_{+}} \backslash\{0,1\}$, then $\lambda$ are multiples of $\alpha$ and we obtain an elegant formula of $\mu_{k_{0}}$ which only contains the terms in $s^{\alpha}$. More generally, if $\alpha$ is rational, then $\mu_{k_{0}}$ contains powers of $s$ of commensurate exponents.

The following example illustrates the case where $\alpha$ is irrational.

\section{Example 1}

$$
G(s)=\left[\frac{e^{-s}}{s^{\pi / 2}\left(s^{\pi / 4}-1\right)^{2}}, \frac{e^{-3 s}}{s^{\pi / 4}-1}\right]
$$

We have $p\left(s^{\pi / 4}\right)=s^{\pi / 2}\left(s^{\pi / 4}-1\right)^{2}$ with degree $d=4$ in $s^{\pi / 4}$. Its unstable roots are $b_{0}=0$ and $b_{1}=1$ with multiplicity $m_{0}=2$ and $m_{1}=2$ respectively. Obviously, $q_{1}\left(s^{\pi / 4}\right)=1$ and $q_{2}\left(s^{\pi / 4}\right)=s^{\pi / 2}\left(s^{\pi / 4}-1\right)$. Then from Proposition 1, we obtain an l.c.f. as follows

$$
\begin{aligned}
& \widetilde{M}(s)=\frac{s^{\pi / 2}\left(s^{\pi / 4}-1\right)^{2}}{\left(s^{\pi / 4}+1\right)^{4}} \\
& \widetilde{N}(s)=\frac{1}{\left(s^{\pi / 4}+1\right)^{4}}\left[e^{-s}, e^{-3 s} s^{\pi / 2}\left(s^{\pi / 4}-1\right)\right] .
\end{aligned}
$$

To complete the expressions of the Bézout factors given in Proposition 2, we now choose $u\left(s^{\pi / 4}\right)$ and search for $\mu_{1}(s)$ and $\mu_{2}(s)$ by solving the equations imposed by the two conditions (5), (6).

Here, we choose $u\left(s^{\pi / 4}\right)=\left(s^{\pi / 4}+1\right)^{4}$. It is easy to see that $k_{0}=1, k_{1}=1, f_{1}=2, f_{2}=0$ and $\mathcal{L}\left(m_{0}, \alpha\right)=$ $\mathcal{L}(2, \pi / 4)=\{0, \pi / 4,1\}$. Therefore, $\mu_{1}(s)$ and $\mu_{2}(s)$ have the forms

$$
\begin{aligned}
\mu_{1}(s)= & \beta_{01}+\beta_{(\pi / 4) 1} s^{\pi / 4}+\beta_{11} s+\beta_{(\pi / 2) 1} s^{\pi / 2} \\
& +\beta_{(3 \pi / 4) 1} s^{3 \pi / 4} \\
\mu_{2}(s)= & 0 .
\end{aligned}
$$

The numerator of $\widetilde{X}(s)$ is then $\left(s^{\pi / 4}+1\right)^{8}-e^{-s} \mu_{1}(s)$.

Its development around zero is

$$
\left(1-\beta_{01}\right)+\left(8-\beta_{(\pi / 4) 1}\right) s^{\pi / 4}+\left(\beta_{01}-\beta_{11}\right) s+O\left(s^{\pi / 2}\right) .
$$

The condition (5) implies that all powers of $s$ with degree smaller than $\pi / 2$ vanish, thus leads to $\beta_{01}=1, \beta_{(\pi / 4) 1}=$ 8 , and $\beta_{11}=1$.

The other coefficients are derived from the condition (6), which is represented by

$$
\begin{aligned}
\left(s^{\pi / 4}+1\right)^{8}-e^{-s} \mu_{1}(s) & =0 \\
{\left[\left(s^{\pi / 4}+1\right)^{8}-e^{-s} \mu_{1}(s)\right]^{\prime} } & =0
\end{aligned}
$$

at $s=1$. The unique solution of these two equations is $\beta_{(\pi / 2) 1}=-2(11 \pi+128 e \pi-2+512 e) / \pi$ and $\beta_{(3 \pi / 4) 1}=$ $4(3 \pi+128 e \pi-1+256 e) / \pi$.

Hence, the Bézout factors are

$$
\begin{aligned}
\tilde{X}(s) & =\frac{\left(s^{\pi / 4}+1\right)^{8}-e^{-s} \mu_{1}(s)}{s^{\pi / 2}\left(s^{\pi / 4}-1\right)^{2}\left(s^{\pi / 4}+1\right)^{4}}, \\
\tilde{Y}(s) & =\left[\frac{\mu_{1}(s)}{\left(s^{\pi / 4}+1\right)^{4}}, 0\right]^{T}
\end{aligned}
$$

where

$$
\begin{aligned}
\mu_{1}(s)= & 1+8 s^{\pi / 4}+s-\frac{2(11 \pi+128 e \pi-2+512 e)}{\pi} s^{\pi / 2} \\
& +\frac{4(3 \pi+128 e \pi-1+256 e)}{\pi} s^{3 \pi / 4}
\end{aligned}
$$

\section{Right coprime factorizations and Bézout fac- tors}

The previous section showed that the systems $G(s)$ under study admit l.c.f.'s over $H_{\infty}$. Since $H_{\infty}$ is a Hermite ring, then there exist r.c.f.'s for $G(s)[19]$.

While l.c.f.'s and left Bézout factors of our transfer matrices are vectors and scalars, the right counterparts are vectors and square matrices with a lot more entries to be determined. Regarding calculation complexity, we separate the study into two classes of systems. The first class consists of systems with distinct unstable poles, i.e. $\widetilde{p}_{k}\left(s^{\alpha}\right)$ and $\widetilde{p}_{k^{\prime}}\left(s^{\alpha}\right)$ have no common unstable roots if $k \neq k^{\prime}$, for which $M(s)$ can be chosen to be of diagonal form. This simple form cannot be applied to the second class of systems with identical unstable poles, for which our first attempt is to consider a particular case.

Some particular cases of the systems considered here have been studied in [12].

\subsection{Distinct poles}

In this context of determining right Bézout factors, the choice of $\mu_{k}$ for $k=1, \ldots, n$ is not very different from that presented in Proposition 2. 
Proposition 3 Let $G(s)$ be given by (1). Suppose that all (zero and non-zero) unstable roots of $\widetilde{p}_{k}\left(s^{\alpha}\right)$ for $k=1, \ldots, n$ are distinct. Then one r.c.f. and associated Bézout factors are given by

$$
\begin{aligned}
& N(s)=\left[N_{1}(s), \ldots, N_{n}(s)\right], \\
& M(s)=\left[\begin{array}{ccc}
M_{11}(s) & \cdots & 0 \\
\vdots & \ddots & \vdots \\
0 & \cdots & M_{n n}(s)
\end{array}\right], \\
& X(s)=\left[\begin{array}{ccc}
X_{11}(s) & \cdots & X_{1 n}(s) \\
\vdots & \ddots & \vdots \\
X_{n 1}(s) & \cdots & X_{n n}(s)
\end{array}\right], \\
& Y(s)=\left[Y_{1}(s), \ldots, Y_{n}(s)\right]^{T},
\end{aligned}
$$

where for $k, k^{\prime} \in\{1, \ldots, n\}$ and $k \neq k^{\prime}$

$$
\begin{aligned}
N_{k}(s) & =\frac{e^{-s h_{k}} \widetilde{q}_{k}\left(s^{\alpha}\right)}{\left(s^{\alpha}+1\right)^{d_{k}}}, \\
M_{k k}(s) & =\frac{\widetilde{p}_{k}\left(s^{\alpha}\right)}{\left(s^{\alpha}+1\right)^{d_{k}}}, \\
Y_{k}(s) & =\frac{\mu_{k}(s)}{u\left(s^{\alpha}\right)} \prod_{1 \leq j \leq n, j \neq k}\left(\left(s^{\alpha}\right)^{m_{0 j}} \prod_{i=1}^{N}\left(s^{\alpha}-b_{i}\right)^{m_{i j}}\right), \\
X_{k k}(s) & =\frac{1-Y_{k}(s) N_{k}(s)}{M_{k k}(s)}, \\
X_{k k^{\prime}}(s) & =-Y_{k}(s) e^{-s h_{k^{\prime}}} \frac{\widetilde{q}_{k^{\prime}}\left(s^{\alpha}\right)}{\widetilde{p}_{k^{\prime}}\left(s^{\alpha}\right)},
\end{aligned}
$$

where $d_{k}$ is the degree of $\widetilde{p}_{k}$ in $s^{\alpha}$; $u$ is a polynomial of degree $d$ in $s^{\alpha}$ that has no unstable zeros; and $\mu_{k}(s)$ have the following form

$\mu_{k}(s)=\sum_{\lambda \in \mathcal{L}\left(m_{0 k}, \alpha\right)} \beta_{\lambda k} s^{\lambda}+\sum_{j=m_{0 k}}^{m_{0 k}+\sum_{i=1}^{N} m_{i k}-1} \beta_{(j \alpha) k}\left(s^{\alpha}\right)^{j}$,

satisfying

$$
\begin{aligned}
& u\left(s^{\alpha}\right)\left(s^{\alpha}+1\right)^{d_{k}}-e^{-s h_{k}} \mu_{k}(s) \widetilde{q}_{k}\left(s^{\alpha}\right) \\
& \times \prod_{1 \leq j \leq n, j \neq k}\left(\prod_{i=1}^{N}\left(s^{\alpha}-b_{i}\right)^{m_{i j}}\right)=O\left(s^{m_{0 k} \alpha}\right)
\end{aligned}
$$

as $s \rightarrow 0$ if $\widetilde{p}_{k}\left(s^{\alpha}\right)$ has a root at zero, and

$$
\begin{aligned}
& {\left[u\left(s^{\alpha}\right)\left(s^{\alpha}+1\right)^{d_{k}}-e^{-s h_{k}} \mu_{k}(s) \widetilde{q}_{k}\left(s^{\alpha}\right)\right.} \\
& \left.\times \prod_{1 \leq j \leq n, j \neq k}\left(\left(s^{\alpha}\right)^{m_{0 j}} \prod_{i=1}^{N}\left(s^{\alpha}-b_{i}\right)^{m_{i j}}\right)\right]^{(l)}=0
\end{aligned}
$$

for each non-zero unstable root of $\widetilde{p}_{k}\left(s^{\alpha}\right)$, i.e. $s=b_{i}^{1 / \alpha}$ with $m_{i k} \neq 0$ for $i=1, \ldots, N$, where $l=0, \ldots, m_{i k}-1$.

PROOF. It is obvious that $N_{k}(s), M_{k k}(s), Y_{k}(s)$, $X_{k k^{\prime}}(s) \in H_{\infty}$. The two conditions (9) and (10) guarantee $X_{k k}(s) \in H_{\infty}$ for $k=1, \ldots, n$. We also see that $G(s)=N(s) M(s)^{-1}$ and the right Bézout identity $X(s) M(s)+Y(s) N(s)=I$ is verified.

To complete the proof, we prove the existence of $\mu_{k}(s)$ satisfying the two conditions in Appendix B.

Here is a numerical example of the use of the proposition.

\section{Example 2}

$$
G(s)=\left[\frac{e^{-s}}{s^{\pi / 2}\left(s^{\pi / 4}-1\right)^{2}}, \frac{e^{-3 s}}{s^{\pi / 4}-2}\right]
$$

By applying Proposition 3 we obtain the r.c.f. as follows

$$
\begin{aligned}
& N(s)=\left[\frac{e^{-s}}{\left(s^{\pi / 4}+1\right)^{4}}, \frac{e^{-3 s}}{s^{\pi / 4}+1}\right], \\
& M(s)=\left[\begin{array}{cc}
\frac{s^{\pi / 2}\left(s^{\pi / 4}-1\right)^{2}}{\left(s^{\pi / 4}+1\right)^{4}} & 0 \\
0 & \frac{s^{\pi / 4}-2}{s^{\pi / 4}+1}
\end{array}\right] .
\end{aligned}
$$

We choose $u\left(s^{\pi / 4}\right)=\left(s^{\pi / 4}+1\right)^{5}$, then $Y(s)$ has the form

$$
Y(s)=\left[\frac{\mu_{1}(s)\left(s^{\pi / 4}-2\right)}{\left(s^{\pi / 4}+1\right)^{5}}, \quad \frac{\mu_{2}(s) s^{\pi / 2}\left(s^{\pi / 4}-1\right)^{2}}{\left(s^{\pi / 4}+1\right)^{5}}\right]^{T}
$$

where $\mu_{1}(s)=\beta_{01}+\beta_{(\pi / 4) 1} s^{\pi / 4}+\beta_{11} s+\beta_{(\pi / 2) 1} s^{\pi / 2}+$ $\beta_{(3 \pi / 4) 1} s^{3 \pi / 4}$ and $\mu_{2}(s)=\beta_{02}$.

The condition (9) is only applied for $k=1$. We develop the numerator of $X_{11}(s)$ around zero as follows

$$
\begin{aligned}
& \left(s^{\pi / 4}+1\right)^{9}-e^{-s} \mu_{1}(s)\left(s^{\pi / 4}-2\right) \\
= & \left(1+2 \beta_{01}\right)+\left(9-\beta_{01}+2 \beta_{(\pi / 4) 1}\right) s^{\pi / 4}+2\left(\beta_{11}-\beta_{01}\right) s \\
& +O\left(s^{\pi / 2}\right) .
\end{aligned}
$$

It turns out that all the terms with order smaller than $\pi / 2$ in the development have to be zero, thus giving $\beta_{01}=$ $-1 / 2, \beta_{(\pi / 4) 1}=-19 / 4$, and $\beta_{11}=-1 / 2$.

Other unknown coefficients are deduced from applying the condition (10). We have

$$
\begin{aligned}
\left(s^{\pi / 4}+1\right)^{9}-e^{-s} \mu_{1}(s)\left(s^{\pi / 4}-2\right) & =0, \\
{\left[\left(s^{\pi / 4}+1\right)^{9}-e^{-s} \mu_{1}(s)\left(s^{\pi / 4}-2\right)\right]^{\prime} } & =0
\end{aligned}
$$


at $s=1$ and

$$
\left(s^{\pi / 4}+1\right)^{6}-e^{-3 s} \mu_{2}(s) s^{\pi / 2}\left(s^{\pi / 4}-1\right)^{2}=0
$$

at $s=2^{4 / \pi}$, and the unique solution of the above equations is $\beta_{(\pi / 2) 1}=(25 \pi+2560 e \pi-4+4096 e) /(2 \pi)$, $\beta_{(3 \pi / 4) 1}=-(27 \pi+7168 e \pi-8+8192 e) /(4 \pi)$, and $\beta_{02}=$ $\left(729\left(e^{2^{4 / \pi}}\right)^{3}\right) / 4$.

$$
\begin{aligned}
& \text { Finally, } X(s)=\left[\begin{array}{ll}
X_{11}(s) & X_{12}(s) \\
X_{21}(s) & X_{22}(s)
\end{array}\right] \text { where } \\
& X_{11}(s)=\frac{\left(s^{\pi / 4}+1\right)^{9}-e^{-s} \mu_{1}(s)\left(s^{\pi / 4}-2\right)}{s^{\pi / 2}\left(s^{\pi / 4}-1\right)^{2}\left(s^{\pi / 4}+1\right)^{5}}, \\
& X_{12}(s)=\frac{-e^{-3 s} \mu_{1}(s)}{\left(s^{\pi / 4}+1\right)^{5}}, \quad X_{21}(s)=\frac{-e^{-s} \mu_{2}(s)}{\left(s^{\pi / 4}+1\right)^{5}}, \\
& X_{22}(s)=\frac{\left(s^{\pi / 4}+1\right)^{6}-e^{-3 s} \mu_{2}(s) s^{\pi / 2}\left(s^{\pi / 4}-1\right)^{2}}{\left(s^{\pi / 4}-2\right)\left(s^{\pi / 4}+1\right)^{5}}, \\
& \mu_{1}(s)=-\frac{1}{2}-\frac{19}{4} s^{\pi / 4}-\frac{1}{2} s \\
& +\frac{25 \pi+2560 e \pi-4+4096 e}{2 \pi} s^{\pi / 2} \\
& -\frac{27 \pi+7168 e \pi-8+8192 e}{4 \pi} s^{3 \pi / 4}, \\
& \mu_{2}(s)=\frac{729\left(e^{2^{4 / \pi}}\right)^{3}}{4} .
\end{aligned}
$$

\subsection{Identical unstable poles}

In the case of identical unstable poles, the following lemma characterizes the form of the matrix $M(s)$.

Lemma 4 Let $G(s)$ be given by (1). Suppose that there exist $k, k^{\prime} \in\{1, \ldots, n\}, k \neq k^{\prime}$ such that $\widetilde{p}_{k}\left(s^{\alpha}\right)$ and $\widetilde{p}_{k^{\prime}}\left(s^{\alpha}\right)$ have common unstable root $(s)$. Then the factor $M(s)$ of an r.c.f. of $G(s)$ cannot be a diagonal matrix.

PROOF. Let us prove the fact by contradiction. Assume that $M(s)$ is diagonal, then $G_{k}(s)=N_{k}(s) / M_{k k}(s)$ $k=1, \ldots, n$. Since $N_{k}(s), M_{k k}(s) \in H_{\infty}$, then the unstable poles of $G_{k}(s)$ are the unstable zeros of $M_{k k}(s)$. Now, from the right Bézout identity $X(s) M(s)+Y(s) N(s)=I$ we obtain for $k, k^{\prime} \in$ $\{1, \ldots, n\}$ and $k \neq k^{\prime}$

$$
\begin{aligned}
X_{k k}(s) & =\frac{1-Y_{k}(s) N_{k}(s)}{M_{k k}(s)}, \\
X_{k k^{\prime}}(s) & =-Y_{k}(s) \frac{N_{k^{\prime}}(s)}{M_{k^{\prime} k^{\prime}}(s)} .
\end{aligned}
$$

Since $X_{k k^{\prime}}(s) \in H_{\infty}$, then all unstable zeros of $M_{k^{\prime} k^{\prime}}(s)$ are zeros of $Y_{k}(s)$. Now, if $G_{k}(s)$ and $G_{k^{\prime}}(s)$ have a common unstable pole $s_{0}$, then $s_{0}$ is a common zero of $M_{k k}(s)$ and $M_{k^{\prime} k^{\prime}}(s)$. Also $Y_{k}\left(s_{0}\right)=0$, and thus $X_{k k}\left(s_{0}\right)=\infty$, which contradicts the fact that $X_{k k}(s) \in H_{\infty}$.

In the following part, we derive r.c.f.'s and Bézout factors for a particular system whose entries (which only have one simple pole) may involve identical poles. To help clarify the demonstration of those results, we will begin with a lemma who derives the inverse of a particular upper triangular matrix.

We consider sparse matrices with the following condition imposed on the entries above the main diagonal: if any entry on the $k$-th column is non-zero, then all other entries on the $k$-th column as well as those on the $k$-th row must be zeros.

Lemma 5 Let $M \in \mathbb{R}^{n \times n}$ be an upper triangular matrix whose entries on the main diagonal are not equal to zero and the entries above the main diagonal satisfy

(A) for $k=1, \ldots, n$, if there exists $l^{\prime \prime} \in \mathbb{Z}, l^{\prime \prime} \in[1, k)$ such that $M_{l^{\prime \prime} k} \neq 0$ then $M_{l k}=0$ for $l \in\{1, \ldots, k-$ $1\} \backslash\left\{l^{\prime \prime}\right\}$ and $M_{k l^{\prime}}=0$ for $l^{\prime} \in\{k+1, \ldots, n\}$.

Then its inverse is given by $M^{-1}=M^{i n v}$, where $M^{\text {inv }}$ is an upper triangular matrix whose entries on and above the main diagonal satisfy

$$
\begin{aligned}
M_{k k}^{i n v} & =\frac{1}{M_{k k}} \\
M_{k k^{\prime}}^{i n v} & =-\frac{M_{k k^{\prime}}}{M_{k k} M_{k^{\prime} k^{\prime}}}
\end{aligned}
$$

for $k, k^{\prime} \in\{1, \ldots, n\}$ and $k<k^{\prime}$.

PROOF. It is obvious that the entries below the main diagonal of the product $M M^{i n v}$ are all zero, and the entries on the main diagonal are all one.

Now we consider the entries above the main diagonal of the product. For $i<j$,

$$
\left(M M^{i n v}\right)_{i j}=\sum_{k=1}^{n} M_{i k} M_{k j}^{i n v}=\sum_{k=i}^{j} M_{i k} M_{k j}^{i n v} .
$$

Considering $i<k<j$, if $M_{i k} \neq 0$, then $M_{k j}=0$ under the assumption (A), and thus $M_{k j}^{i n v}=0$ due to (12). Therefore,

$$
\left(M M^{i n v}\right)_{i j}=M_{i i} M_{i j}^{i n v}+M_{i j} M_{j j}^{i n v} .
$$

By replacing $M_{i j}^{i n v}$ and $M_{j j}^{i n v}$ with (12) and (11) respectively, we obtain $\left(M M^{i n v}\right)_{i j}=0$. 


\section{Example 3}

$$
M=\left[\begin{array}{ccccc}
M_{11} & 0 & M_{13} & 0 & 0 \\
0 & M_{22} & 0 & M_{24} & M_{25} \\
0 & 0 & M_{33} & 0 & 0 \\
0 & 0 & 0 & M_{44} & 0 \\
0 & 0 & 0 & 0 & M_{55}
\end{array}\right]
$$

with $M_{i i} \neq 0, i=1, \ldots, 5$, and $M_{13}, M_{24}, M_{25} \neq 0$. This matrix satisfies all the conditions in Lemma 5 and thus its inverse is

$$
M^{-1}=\left[\begin{array}{ccccc}
\frac{1}{M_{11}} & 0 & -\frac{M_{13}}{M_{11} M_{33}} & 0 & 0 \\
0 & \frac{1}{M_{22}} & 0 & -\frac{M_{24}}{M_{22} M_{44}} & -\frac{M_{25}}{M_{22} M_{55}} \\
0 & 0 & \frac{1}{M_{33}} & 0 & 0 \\
0 & 0 & 0 & \frac{1}{M_{44}} & 0 \\
0 & 0 & 0 & 0 & \frac{1}{M_{55}}
\end{array}\right] .
$$

In the following proposition, we consider $G(s)$ with one pole for each of its entries and some entries may have the same pole. To simplify the presentation, we assume that the delays are ordered. A discussion on how to apply the next result to the case of unordered delays will follow the proposition.

Proposition 6 Let $G(s)$ be given by (1) with $h_{1} \leq \ldots \leq$ $h_{n}$ and

$$
R_{k}\left(s^{\alpha}\right)=\frac{a_{k}}{s^{\alpha}-\sigma_{k}},
$$

where $a_{k}, \sigma_{k} \in \mathbb{R}$ for $k=1, \ldots, n$. We denote $\mathcal{I}_{1}:=\emptyset$ and $\mathcal{I}_{k}:=\left\{j \mid j \in\{1, \ldots, k-1\}, \sigma_{j}=\sigma_{k}\right\}$ for $k=2, \ldots, n$. One r.c.f. and associated Bézout factors are given by

$$
\begin{aligned}
N(s) & =\left[N_{1}(s), \ldots, N_{n}(s)\right], \\
M(s) & =\left[\begin{array}{ccc}
M_{11}(s) & \cdots & M_{1 n}(s) \\
\vdots & \ddots & \vdots \\
0 & \cdots & M_{n n}(s)
\end{array}\right], \\
Y(s) & =\left[Y_{1}(s), \ldots, Y_{n}(s)\right]^{T}, \\
X(s) & =M^{-1}(s)-Y(s) G(s),
\end{aligned}
$$

where for $k, k^{\prime} \in\{1, \ldots, n\}$ and $k^{\prime} \neq k$

$$
N_{k}(s)= \begin{cases}0 & \text { if } \mathcal{I}_{k} \neq \emptyset \\ \frac{a_{k} e^{-s h_{k}}}{s^{\alpha}+1} & \text { otherwise }\end{cases}
$$

$$
\begin{aligned}
M_{k k}(s) & = \begin{cases}1 & \text { if } \mathcal{I}_{k} \neq \emptyset \\
\frac{s^{\alpha}-\sigma_{k}}{s^{\alpha}+1} & \text { otherwise, }\end{cases} \\
M_{k^{\prime} k}(s) & = \begin{cases}-\frac{a_{k} e^{-s\left(h_{k}-h_{k^{\prime}}\right)}}{a_{k^{\prime}}} & \text { if } k^{\prime}=\min \mathcal{I}_{k}, \\
0 & \text { otherwise, }\end{cases} \\
Y_{k}(s) & = \begin{cases}0 & \text { if } \mathcal{I}_{k} \neq \emptyset, \\
\frac{\beta_{k} \hat{p}_{k}\left(s^{\alpha}\right)}{u\left(s^{\alpha}\right)} & \text { otherwise },\end{cases}
\end{aligned}
$$

where $u\left(s^{\alpha}\right)$ is a polynomial of degree $d$ in $s^{\alpha}$ that has no unstable zeros; $\hat{p}_{k}\left(s^{\alpha}\right)=\frac{p\left(s^{\alpha}\right)}{s^{\alpha}-\sigma_{k}}$; the coefficients $\beta_{k}$, for those $k$ such that $\mathcal{I}_{k}=\emptyset$ and $\sigma_{k} \geq 0$, are given by

$$
\beta_{k}=\frac{u\left(\sigma_{k}\right)\left(\sigma_{k}+1\right) e^{\sigma_{k}^{1 / \alpha} h_{k}}}{a_{k} \hat{p}_{k}\left(\sigma_{k}\right)},
$$

while $\beta_{k}$ for other $k$ can be chosen arbitrarily, and $M^{-1}(s)=M^{i n v}(s)$, where $M^{i n v}(s)$ is an upper triangular matrix whose entries on and above the main diagonal are given by

$$
\begin{aligned}
M_{k k}^{i n v}(s) & =\frac{1}{M_{k k}(s)}, \\
M_{k k^{\prime}}^{i n v}(s) & =-\frac{M_{k k^{\prime}}(s)}{M_{k k}(s) M_{k^{\prime} k^{\prime}}(s)}
\end{aligned}
$$

for $k, k^{\prime} \in\{1, \ldots, n\}$ and $k<k^{\prime}$.

PROOF. Let us prove that $M(s)$ given by (14), (16), and (17) satisfies the assumptions in Lemma 5. Let $k \in$ $\{1, \ldots, n\}$, if $M_{k^{\prime} k}(s) \neq 0$ then due to (17) $k^{\prime}=\min \mathcal{I}_{k}$, and $M_{k^{\prime \prime} k}(s)=0$ for $k^{\prime \prime} \neq k^{\prime}$. Also, since $k^{\prime}=\min \mathcal{I}_{k}$, then $k \neq \min \mathcal{I}_{k^{\prime \prime}}$ for $k^{\prime \prime}>k$, and thus $M_{k k^{\prime \prime}}(s)=0$. Hence the assumption (A) in Lemma 5 is satisfied.

Consequently, due to Lemma 5 , the inverse of $M(s)$ is $M^{i n v}(s)$ whose entries are given by (19).

We now prove that $N(s) M^{-1}(s)=G(s)$.

For $k \in\{1, \ldots, n\}$, we have

$$
\left(N(s) M(s)^{-1}\right)_{k}=\sum_{l=1}^{n} N_{l}(s) M_{l k}^{i n v}(s)=\sum_{l=1}^{k} N_{l}(s) M_{l k}^{i n v}(s)
$$

- If $\mathcal{I}_{k}=\emptyset$, then $M_{l k}^{\text {inv }}(s)=0$ for $l=1, \ldots, k-1$, and $\left(N(s) M(s)^{-1}\right)_{k}=N_{k}(s) M_{k k}^{i n v}(s)=e^{-s h_{k}} R_{k}\left(s^{\alpha}\right)$.

- If $\mathcal{I}_{k} \neq \emptyset$, then $M_{l k}^{\text {inv }}(s)=0$ for $l \in\{1, \ldots, k-1\} \backslash\left\{k^{\prime}\right\}$ where $k^{\prime}=\min \mathcal{I}_{k}$ and $N_{k}(s)=0$. Therefore,

$$
\begin{aligned}
\left(N(s) M(s)^{-1}\right)_{k} & =N_{k^{\prime}}(s) M_{k^{\prime} k}^{i n v}(s) \\
& =-N_{k^{\prime}}(s) \frac{M_{k^{\prime} k}(s)}{M_{k^{\prime} k^{\prime}}(s) M_{k k}(s)} .
\end{aligned}
$$


Note that $\mathcal{I}_{k^{\prime}}=\emptyset$ since $k^{\prime}=\min \mathcal{I}_{k}$. By replacing the above terms with appropriate expressions in (15), (16) and (17) and by noting that $\sigma_{k^{\prime}}=\sigma_{k}$, we get $\left(N(s) M(s)^{-1}\right)_{k}=e^{-s h_{k}} R_{k}\left(s^{\alpha}\right)$.

It is obvious that $N_{k}(s), M_{k k}(s), M_{k^{\prime} k}(s), Y_{k}(s) \in H_{\infty}$. Let us now prove that $X(s) \in \mathbf{M}\left(H_{\infty}\right)$.

For $k, k^{\prime} \in\{1, \ldots, n\}$, we have $X_{k^{\prime} k}(s)=M_{k^{\prime} k}^{i n v}(s)-$ $Y_{k^{\prime}}(s) e^{-s h_{k}} R_{k}\left(s^{\alpha}\right)$.

- If $\mathcal{I}_{k^{\prime}} \neq \emptyset$, then $Y_{k^{\prime}}(s)=0$, and thus $X_{k^{\prime} k}(s)=$ $M_{k^{\prime} k}^{i n v}(s)$. Now, for $k^{\prime}>k, M_{k^{\prime} k}^{\text {inv }}(s)=0$. For $k^{\prime}=k$, $M_{k^{\prime} k}^{i n v}(s)=1$. For $k^{\prime}<k$, from the fact that $\mathcal{I}_{k^{\prime}} \neq \emptyset$, we deduce that $k^{\prime} \neq \min \mathcal{I}_{k}$, thus $M_{k^{\prime} k}^{\text {inv }}(s)=0$.

- If $\mathcal{I}_{k^{\prime}}=\emptyset$, then $Y_{k^{\prime}}(s)$ involves $\hat{p}_{k^{\prime}}\left(s^{\alpha}\right)$.

For $k^{\prime}>k$, the fact that $\mathcal{I}_{k^{\prime}}=\emptyset$ leads to $\sigma_{k^{\prime}} \neq \sigma_{k}$. Therefore,

$$
Y_{k^{\prime}}(s) R_{k}\left(s^{\alpha}\right)=\frac{a_{k} \beta_{k^{\prime}} p\left(s^{\alpha}\right)}{\left(s^{\alpha}-\sigma_{k}\right)\left(s^{\alpha}-\sigma_{k^{\prime}}\right) u\left(s^{\alpha}\right)}
$$

belongs to $H_{\infty}$ since $\left(s^{\alpha}-\sigma_{k}\right)\left(s^{\alpha}-\sigma_{k^{\prime}}\right)$ is eliminated by the same term in $p\left(s^{\alpha}\right)$. It is also obvious that $M_{k^{\prime} k}^{i n v}(s)=0$. Therefore, $X_{k^{\prime} k}(s) \in H_{\infty}$.

For $k^{\prime}=k$, we have

$$
X_{k^{\prime} k^{\prime}}(s)=\frac{u\left(s^{\alpha}\right)\left(s^{\alpha}+1\right)-\beta_{k^{\prime}} \hat{p}_{k^{\prime}}\left(s^{\alpha}\right) a_{k^{\prime}} e^{-s h_{k^{\prime}}}}{u\left(s^{\alpha}\right)\left(s^{\alpha}-\sigma_{k^{\prime}}\right)} .
$$

If $\sigma_{k^{\prime}}<0$, then $X_{k^{\prime} k^{\prime}}(s) \in H_{\infty}$ for all $\beta_{k^{\prime}}$. If $\sigma_{k^{\prime}} \geq 0$, since $\beta_{k^{\prime}}$ given by (18) makes the numerator of $X_{k^{\prime} k^{\prime}}(s)$ vanish at $s=\sigma_{k^{\prime}}^{1 / \alpha}$, then $X_{k^{\prime} k^{\prime}}(s) \in H_{\infty}$.

For $k^{\prime}<k$, suppose first that $k^{\prime} \neq \min \mathcal{I}_{k}$. From this fact together with $\mathcal{I}_{k^{\prime}}=\emptyset$ we deduce that $\sigma_{k^{\prime}} \neq \sigma_{k}$, and thus $Y_{k^{\prime}}(s) R_{k}\left(s^{\alpha}\right) \in H_{\infty}$. We also have $M_{k^{\prime} k}^{i n v}(s)=0$, leading to $X_{k^{\prime} k}(s) \in H_{\infty}$. In the case where $k^{\prime}=\min \mathcal{I}_{k}$, we have $\sigma_{k^{\prime}}=\sigma_{k}$ and

$$
X_{k^{\prime} k}(s)=a_{k} e^{-s h_{k}} \frac{u\left(s^{\alpha}\right)\left(s^{\alpha}+1\right) e^{s h_{k^{\prime}}}-\beta_{k^{\prime}} \hat{p}_{k^{\prime}}\left(s^{\alpha}\right) a_{k^{\prime}}}{u\left(s^{\alpha}\right)\left(s^{\alpha}-\sigma_{k^{\prime}}\right) a_{k^{\prime}}} .
$$

By the same argument as in the case where $k^{\prime}=k$, we conclude that $X_{k^{\prime} k}(s) \in H_{\infty}$.

The right Bézout identity $X(s) M(s)+Y(s) N(s)=I$ is clearly satisfied.

Remark 3 A transfer matrix $G$ given by (1) with the delays of its elements not in order can be transformed to a transfer matrix $G_{0}$ with ordered delays by multiplying $G$ by an appropriate permutation matrix $P$. It is well known that this matrix $P$ is orthogonal and its inverse is $P^{T}$. Assume that $\left(M_{0}, N_{0}\right)$ is an r.c.f. over $H_{\infty}$ of $G_{0}$ and $X_{0}, Y_{0}$ are the corresponding right Bézout factors. We have then $G=G_{0} P^{-1}=N_{0} M_{0}^{-1} P^{-1}=$
$N_{0}\left(P M_{0}\right)^{-1}$. It is obvious that $P M_{0} \in \mathbf{M}\left(H_{\infty}\right)$. Furthermore, $X_{0} P^{-1} P M_{0}+Y_{0} N_{0}=I$ and $X_{0} P^{-1} \in \mathbf{M}\left(H_{\infty}\right)$. Hence, $\left(P M_{0}, N_{0}\right)$ is an r.c.f. of $G$ and $X_{0} P^{-1}, Y_{0}$ are the corresponding Bézout factors.

The next example illustrates the proposition.

\section{Example 4}

$$
G(s)=\left[\frac{e^{-s}}{\sqrt{s}}, \frac{e^{-s}}{\sqrt{s}-1}, \frac{e^{-3 s}}{\sqrt{s}-1}\right]
$$

From (13), (14), (15), (16), and (17), we obtain

$$
\begin{aligned}
& N(s)=\left[\frac{e^{-s}}{\sqrt{s}+1}, \frac{e^{-s}}{\sqrt{s}+1}, 0\right], \\
& M(s)=\left[\begin{array}{ccc}
\frac{\sqrt{s}}{\sqrt{s}+1} & 0 & 0 \\
0 & \frac{\sqrt{s}-1}{\sqrt{s}+1}-e^{-2 s} \\
0 & 0 & 1
\end{array}\right] .
\end{aligned}
$$

The inverse of $M(s)$ is

$$
M^{-1}(s)=\left[\begin{array}{ccc}
\frac{\sqrt{s}+1}{\sqrt{s}} & 0 & 0 \\
0 & \frac{\sqrt{s}+1}{\sqrt{s}-1} & \frac{e^{-2 s}(\sqrt{s}+1)}{\sqrt{s}-1} \\
0 & 0 & 1
\end{array}\right] \text {. }
$$

The least common denominator of the entries of $G(s)$ is $p(\sqrt{s})=\sqrt{s}(\sqrt{s}-1)$. Then $\hat{p}_{1}(\sqrt{s})=\sqrt{s}-1$, and $\hat{p}_{2}(\sqrt{s})=\sqrt{s}$. We choose $u(\sqrt{s})=(\sqrt{s}+1)^{2}$, which has no unstable roots. We have then

$Y(s)=\left[\frac{\beta_{1}(\sqrt{s}-1)}{(\sqrt{s}+1)^{2}}, \frac{\beta_{2} \sqrt{s}}{(\sqrt{s}+1)^{2}}, 0\right]^{T}$,
$X(s)=M^{-1}(s)-Y(s) G(s)=\left[\begin{array}{ccc}X_{11}(s) & X_{12}(s) & X_{13}(s) \\ X_{21}(s) & X_{22}(s) & X_{23}(s) \\ 0 & 0 & 1\end{array}\right]$,

where

$$
\begin{aligned}
& X_{11}(s)=\frac{(\sqrt{s}+1)^{3}-\beta_{1}(\sqrt{s}-1) e^{-s}}{\sqrt{s}(\sqrt{s}+1)^{2}}, \\
& X_{12}(s)=-\frac{\beta_{1} e^{-s}}{(\sqrt{s}+1)^{2}}, \quad X_{13}(s)=-\frac{\beta_{1} e^{-3 s}}{(\sqrt{s}+1)^{2}}, \\
& X_{21}(s)=-\frac{\beta_{2} e^{-s}}{(\sqrt{s}+1)^{2}}, \\
& X_{22}(s)=\frac{(\sqrt{s}+1)^{3}-\beta_{2} e^{-s} \sqrt{s}}{(\sqrt{s}-1)(\sqrt{s}+1)^{2}} \\
& X_{23}(s)=\frac{e^{-2 s}(\sqrt{s}+1)^{3}-\beta_{2} e^{-3 s} \sqrt{s}}{(\sqrt{s}-1)(\sqrt{s}+1)^{2}} .
\end{aligned}
$$


We see that $X_{12}(s), X_{13}(s), X_{21}(s) \in H_{\infty}$. From (18), we obtain $\beta_{1}=-1$ and $\beta_{2}=8 e$, which make $X_{11}(s)$, $X_{22}(s)$, and $X_{23}(s)$ be in $H_{\infty}$ respectively.

\section{Conclusion}

In this paper, we have considered MISO fractional systems with input or output delays. Explicit expressions of an l.c.f. over $H_{\infty}$ of the transfer matrices as well as the corresponding Bézout factors are given. Right coprime factorizations and right Bézout factors are also found for systems with entries of the transfer matrix containing different poles. In the case of identical poles, the right factors are primarily found for some simple classes of systems. Hence, in conclusion, we can have Youla-Kučera parametrization of stabilizing controllers for all systems with distinct unstable poles and a class of systems with identical unstable poles where each element of the transfer matrix involves one pole since for these systems both l.c.f.'s and r.c.f.'s and Bézout factors are available.

Even if similar techniques may in principle be used for MIMO systems, the analysis appears to be much more involved and requires a full separate study.

The proposed controllers in this paper given in terms of proper transfer functions containing only standard polynomials, fractional polynomials and delays are realizable. Their practical implementation is the objective of a forthcoming work.

\section{References}

[1] C. Bonnet and J. R. Partington. Analysis of fractional delay systems of retarded and neutral type. Automatica, 38(7):1133-1138, 2002.

[2] C. Bonnet and J. R. Partington. PID stabilization of SISO delay systems and robust stabilization of systems with multiple transmission delays. In 16th International Symposium on Mathematical Theory of Networks and Systems, pages 1-7, 2004.

[3] C. Bonnet and J. R. Partington. Stabilization of some fractional delay systems of neutral type. Automatica, 43(12):2047-2053, 2007.

[4] R. F. Curtain, G. Weiss, and M. Weiss. Coprime factorization for regular linear systems. Automatica, 32(11):1519-1531, 1996.

[5] T.J. Freeborn. A survey of fractional-order circuit models for biology and biomedicine. IEEE Journal on Emerging and Selected Topics in Circuits and Systems, 3(3):416-424, 2013.

[6] S. E. Hamamci. An algorithm for stabilization of fractional-order time delay systems using fractional-order PID controllers. IEEE Transactions on Automatic Control, 52(10):1964-1969, 2007.

[7] K. S. Miller and B. Ross. An Introduction to the Fractional Calculus and Fractional Differential Equations. Wiley, 1993.

[8] L. Mirkin and N. Raskin. State-space parametrization of all stabilizing dead-time controllers. In 38th IEEE Conference on Decision and Control, volume 1, pages 221-226, 1999.
[9] A. A. Moelja and G. Meinsma. Parametrization of stabilizing controllers for systems with multiple I/O delays. In 4th IFAC Workshop on Time Delay Systems, 2003.

[10] S. Mondié and J. J. Loiseau. Finite eigenstructure assignment for input delay systems. In S.-I. Niculescu and K. Gu, editors, Advances in Time-Delay Systems. Springer, 2004.

[11] L. H. V. Nguyen and C. Bonnet. Coprime factorizations of MISO fractional time-delay systems. In 20th International Symposium on Mathematical Theory of Networks and Systems, July 2012.

[12] L. H. V. Nguyen and C. Bonnet. Right coprime factorizations of MISO fractional time-delay systems. In 1st IFAC Workshop on Control of Systems Modeled by Partial Differential Equations, September 2013.

[13] H. Özbay, C. Bonnet, and A. R. Fioravanti. PID controller design for fractional-order systems with time delays. Systems and Control Letters, 61(1):18-23, 2012.

[14] A. Quadrat. On a generalization of the Youla-Kučera parametrization, Part II: The lattice approach to MIMO systems. Mathematics of Control, Signals, and Systems, 18(3):199-235, 2006.

[15] P. F. Quet, B. Ataşlar, A. İftar, H. Özbay, S. Kalyanaraman, and T. Kang. Rate-based flow controllers for communication networks in the presence of uncertain time-varying multiple time-delays. Automatica, 38(6):917-928, 2002.

[16] J.-P. Richard. Time-delay systems: an overview of some recent advances and open problems. Automatica, 39:16671694, 2003.

[17] J. Sabatier, O. P. Agrawal, and J. A. Tenreiro Machado. Advances in Fractional Calculus: Theoretical Developments and Applications in Physics and Engineering. Springer, 2007.

[18] A. Si-Ammour, S. Djennoune, and M. Bettayeb. A sliding mode control for linear fractional systems with input and state delays. Communications in Nonlinear Science and Numerical Simulation, 14(5):2310 - 2318, 2009.

[19] M. C. Smith. On stabilization and the existence of coprime factorizations. IEEE Transactions on Automatic Control, 34(9):1005-1007, 1989.

[20] M. Vidyasagar. Control System Synthesis: A Factorization Approach. MIT Press, 1985.

\section{A Proof of Proposition 2}

First, we consider the condition (5) on the poles at zero. If the system has no zero pole, then the condition is satisfied. Otherwise, the condition imposes that all powers of $s$ whose order is smaller than $m_{0} \alpha$ are eliminated in the development of the numerator of $\widetilde{X}(s)$ around zero. When $s \rightarrow 0$, we have

$$
\begin{aligned}
\left(s^{\alpha}+1\right)^{d} u\left(s^{\alpha}\right) & =\sum_{\lambda \in \mathcal{L}\left(m_{0}, \alpha\right)} a_{\lambda} s^{\lambda}+O\left(s^{m_{0} \alpha}\right), \\
e^{-s h_{k}} q_{k}\left(s^{\alpha}\right) & =\sum_{\lambda \in \mathcal{L}\left(m_{0}, \alpha\right)} \gamma_{\lambda k} s^{\lambda}+O\left(s^{m_{0} \alpha}\right), \\
\mu_{k}(s) & =\sum_{\lambda \in \mathcal{L}\left(m_{0}, \alpha\right)} \beta_{\lambda k} s^{\lambda}+O\left(s^{m_{0} \alpha}\right) .
\end{aligned}
$$


Then the development of the numerator of $\widetilde{X}(s)$ around zero can be represented in matrix form as follows

$$
\begin{aligned}
& \left(s^{\alpha}+1\right)^{d} u\left(s^{\alpha}\right)-\sum_{k=1}^{n} e^{-s h_{k}} q_{k}\left(s^{\alpha}\right) \mu_{k}(s) \\
= & {\left[s^{\lambda_{N^{\prime \prime}-1}}, s^{\lambda_{N^{\prime \prime}-2}}, \ldots, s^{\lambda_{0}}\right]\left(A-\sum_{k=1}^{n} \Gamma_{k} B_{k}\right)+O\left(s^{m_{0} \alpha}\right), }
\end{aligned}
$$

where $N^{\prime \prime}=\operatorname{card}\left(\mathcal{L}\left(m_{0}, \alpha\right)\right)$ and $0=\lambda_{0}<\ldots<$ $\lambda_{N^{\prime \prime}-1} \in \mathcal{L}\left(m_{0}, \alpha\right), A=\left[a_{\lambda_{N^{\prime \prime}-1}}, \ldots, a_{\lambda_{0}}\right]^{T}, B_{k}=$ $\left[\beta_{\lambda_{N^{\prime \prime}-1} k}, \ldots, \beta_{\lambda_{0} k}\right]^{T}$, and $\Gamma_{k} \in \mathbb{R}^{N^{\prime \prime} \times N^{\prime \prime}}$ are upper triangular matrices which contain $\gamma_{\lambda k}$ and whose entries on the main diagonal are all $\gamma_{\lambda_{0} k}$. The condition (5) is then equivalent to $\sum_{k=1}^{n} \Gamma_{k} B_{k}=A$. For $k=k_{0}$ with $k_{0}$ defined by $(3)$, since $q_{k_{0}}\left(s^{\alpha}\right)$ does not have roots at zero, then (A.1) can be used and implies that $\gamma_{\lambda_{0} k_{0}} \neq 0$. Hence $\operatorname{det} \Gamma_{k_{0}} \neq 0$ and thus $B_{k_{0}}$ admits a unique solution for any values of $\beta_{\lambda k}$ with $\lambda<m_{0} \alpha$ and $k \in\{1, \ldots, n\}, k \neq k_{0}$.

Next, we analyze the condition (6) on non-zero poles.

We first examine the system of equations obtained by replacing $s$ by a non-zero unstable pole $b_{i}^{1 / \alpha}$ and study the existence of $\mu_{k_{i}}^{(l)}\left(b_{i}^{1 / \alpha}\right)$, where $k_{i}$ is defined by (4) and $l=$ $0, \ldots, m_{i}-1$, satisfying the equations. The first equation which corresponds to $l=0$ contains $q_{k_{i}}\left(b_{i}\right) \mu_{k_{i}}\left(b_{i}^{1 / \alpha}\right)$. The second equation, i.e. $l=1$, contains a linear sum of $q_{k_{i}}\left(b_{i}\right) \mu_{k_{i}}\left(b_{i}^{1 / \alpha}\right)$ and $q_{k_{i}}\left(b_{i}\right) \mu_{k_{i}}^{\prime}\left(b_{i}^{1 / \alpha}\right)$. Generally, the equation corresponding to the $l$-th derivative contains a linear sum of $q_{k_{i}}\left(b_{i}\right) \mu_{k_{i}}^{\left(l_{2}\right)}\left(b_{i}^{1 / \alpha}\right)$ with $l_{2}=0, \ldots, l-1$. Since $q_{k_{i}}\left(b_{i}\right) \neq 0$, the system of $m_{i}$ equations can be recursively solved for $m_{i}$ unknowns $\mu_{k_{i}}^{(l)}\left(b_{i}^{1 / \alpha}\right)$ with $l=$ $0, \ldots, m_{i}-1$ for arbitrary values of $\mu_{k}^{(l)}\left(b_{i}^{1 / \alpha}\right)$ for $k=$ $1, \ldots, n, k \neq k_{i}$ and $l=0, \ldots, m_{i}-1$ and admits a unique solution.

Hence, for each $k \in\{1, \ldots, n\}$ and $k \neq k_{0}$, the coefficients $\beta_{(j \alpha) k}, j=0, \ldots, f_{k}-1$ of $\mu_{k}(s)$ satisfy $\mu_{k}^{(l)}\left(b_{i}^{1 / \alpha}\right)=a_{k, i, l}$ for $i=1, \ldots, N$ such that $k_{i}=k$ and $l=0, \ldots, m_{i}-1$. This is the problem of Hermite interpolation and there exists a unique solution.

For $k=k_{0}$, the coefficients of $\mu_{k_{0}}(s)$, i.e. $\beta_{\lambda k_{0}}$ with $\lambda \in \mathcal{L}\left(m_{0}, \alpha\right)$ and $\beta_{(j \alpha) k_{0}}$ with $j=m_{0}, \ldots, m_{0}+f_{k_{0}}-1$, satisfy the equations $\mu_{k_{0}}^{(l)}\left(b_{i}^{1 / \alpha}\right)=a_{k_{0}, i, l}$ for $i=$ $1, \ldots, N$ such that $k_{i} \stackrel{k_{0}}{=} k_{0}$ and $l=0, \ldots, m_{i}-$ 1. We can write $\mu_{k_{0}}(s)=\nu_{k_{0}}(s)+s^{m_{0} \alpha} \eta_{k_{0}}\left(s^{\alpha}\right)$, where $\nu_{k_{0}}(s)=\sum_{\lambda \in \mathcal{L}\left(m_{0}, \alpha\right)} \beta_{\lambda k_{0}} s^{\lambda}$ and $\eta_{k_{0}}\left(s^{\alpha}\right)=$ $\sum_{j=m_{0}}^{m_{0}+f_{k_{0}}-1} \beta_{(j \alpha) k_{0}} s^{\left(j-m_{0}\right) \alpha}$. For arbitrary values of the coefficients $\beta_{\lambda k_{0}}$ with $\lambda \in \mathcal{L}\left(m_{0}, \alpha\right)$, we can derive the values of $\eta_{k_{0}}^{(l)}\left(b_{i}\right)$ for $i=1, \ldots, N$ such that $k_{i}=k_{0}$ and $l=0, \ldots, m_{i}-1$. Note that the numbers of unknowns and of equations are the same and are equal to $f_{k_{0}}$. This returns to the problem of Hermite interpolation and there exists a unique solution.

\section{B Proof of Proposition 3}

First, we consider the condition (9) on zero pole. For $k \in\{1, \ldots, n\}$ such that $m_{0 k}>0$, we develop the terms of the numerator of $X_{k k}(s)$ around zero as follows

$$
\begin{aligned}
& u\left(s^{\alpha}\right)\left(s^{\alpha}+1\right)^{d_{k}}=\sum_{\lambda \in \mathcal{L}\left(m_{0 k}, \alpha\right)} a_{\lambda} s^{\lambda}+O\left(s^{m_{0 k} \alpha}\right), \\
& \mu_{k}(s)=\sum_{\lambda \in \mathcal{L}\left(m_{0 k}, \alpha\right)} \beta_{\lambda k} s^{\lambda}+O\left(s^{m_{0 k} \alpha}\right), \\
& e^{-s h_{k}} \widetilde{q}_{k}\left(s^{\alpha}\right) \prod_{1 \leq j \leq n, j \neq k}\left(\prod_{i=1}^{N}\left(s^{\alpha}-b_{i}\right)^{m_{i j}}\right) \\
&=\sum_{\lambda \in \mathcal{L}\left(m_{0 k}, \alpha\right)} \gamma_{\lambda k} s^{\lambda}+O\left(s^{m_{0 k} \alpha}\right) .
\end{aligned}
$$

Then the development of the numerator of $X_{k k}(s)$ when $s \rightarrow 0$ can be written as

$$
\begin{aligned}
& u\left(s^{\alpha}\right)\left(s^{\alpha}+1\right)^{d_{k}}-e^{-s h_{k}} \mu_{k}(s) \widetilde{q}_{k}\left(s^{\alpha}\right) \\
& \times \prod_{1 \leq j \leq n, j \neq k}\left(\prod_{i=1}^{N}\left(s^{\alpha}-b_{i}\right)^{m_{i j}}\right) \\
& =\left[s^{\lambda_{N_{k}^{\prime \prime}-1}}, s^{\lambda_{N_{k}^{\prime \prime}-2}}, \ldots, s^{\lambda_{0}}\right]\left(A_{k}-\Gamma_{k} B_{k}\right)+O\left(s^{m_{0 k} \alpha}\right),
\end{aligned}
$$

where $N_{k}^{\prime \prime}=\operatorname{card}\left(\mathcal{L}\left(m_{0 k}, \alpha\right)\right), \quad \lambda_{j} \in \mathcal{L}\left(m_{0 k}, \alpha\right)$, $0=\lambda_{0}<\ldots<\lambda_{N_{k}^{\prime \prime}-1} ; A_{k}=\left[a_{N_{k}^{\prime \prime}-1}, \ldots, a_{0}\right]^{T} ;$ $B_{k}=\left[\beta_{\lambda_{N_{k}^{\prime \prime}-1} k}, \ldots, \beta_{\lambda_{0} k}\right]^{T}$; and $\Gamma_{k}$ is an upper triangular matrix whose entries on the main diagonal are all $\gamma_{\lambda_{0} k}$. The condition (9) implies the elimination of the powers of $s$ with order smaller than $m_{0 k} \alpha$ in the numerator, which leads to $\Gamma_{k} B_{k}=A_{k}$. From (B.1), we see that $\gamma_{\lambda_{0} k} \neq 0$, which implies $\Gamma_{k}$ is invertible and $B_{k}$ admits a unique solution.

Now, we analyze the second condition (10) related to non-zero unstable poles. We can write $\mu_{k}(s)=$ $\nu_{k}(s)+s^{m_{0 k} \alpha} \eta_{k}\left(s^{\alpha}\right)$, where $\nu_{k}(s)=\sum_{\lambda \in \mathcal{L}\left(m_{0 k}, \alpha\right)} \beta_{\lambda k} s^{\lambda}$ and $\eta_{k}\left(s^{\alpha}\right)=\sum_{j=m_{0 k}}^{m_{0 k}+\sum_{i=1}^{N} m_{i k}-1} \beta_{(j \alpha) k} s^{\left(j-m_{0 k}\right) \alpha}$. Then for each $k \in\{1, \ldots, n\}$, we can derive the values of $\eta_{k}^{(l)}\left(b_{i}\right)$ for $i \in\{1, \ldots, N\}$ such that $m_{i k}>0$ and $l=0, \ldots, m_{i k}-1$. If we consider the coefficients $\beta_{(j \alpha) k}$, $j=m_{0 k}, \ldots, m_{0 k}+\sum_{i=1}^{N} m_{i k}-1$ as unknowns, then the number of unknowns is the same as the number of equations and is equal to $\sum_{i=1}^{N} m_{i k}$. This is the problem of Hermite interpolation and there exists a unique solution. 